\title{
Revisiting Fitch and Hauser's Observation That Tamarin Monkeys Can Learn Combinations Based on Finite-State Grammar
}

\author{
Shigeru Miyagawa* \\ Department of Linguistics and Philosophy, Massachusetts Institute of Technology, Cambridge, MA, United States
}

Keywords: Chomsky hierarchy, tamarin, finite-state grammar, phrase-structure grammar, alarm calls, frontal operculum, Broca's area, putty-nosed monkey

\section{INTRODUCTION}

In a groundbreaking work, Fitch and Hauser (2004) compared artificial grammar learning between human and cotton-top tamarins (Saguinus oedipus) using finite-state grammar (FSG) and phrasestructure grammar (PSG) types. They found that while humans are able to learn both grammar types, the tamarin monkeys could only learn combinations based on FSG. FSGs process linearly ordered strings whose structure resorts to strictly adjacent steps. Examples of FSGs are $\mathrm{A}^{\mathrm{n}}$ and $(\mathrm{AB})^{\mathrm{n}}$, where $n$ indicates the number of times $\mathrm{A}$ and $\mathrm{AB}$ are repeated, and $\mathrm{A}^{\mathrm{n}} \mathrm{B}^{\mathrm{m}}$, where $n \neq m$. On the other hand, PSGs are not limited to adjacency. This allows PSGs to match the number of

\section{OPEN ACCESS}

Edited by:

Dieter Hillert,

San Diego State University,

United States

Reviewed by:

Drew Rendall,

University of New Brunswick

Fredericton, Canada

*Correspondence:

Shigeru Miyagawa

miyagawa@mit.edu

Specialty section:

This article was submitted to

Language Sciences,

a section of the journal

Frontiers in Psychology

Received: 07 September 2021 Accepted: 02 November 2021 Published: 29 November 2021

Citation:

Miyagawa S (2021) Revisiting Fitch and Hauser's Observation That

Tamarin Monkeys Can Learn Combinations Based on Finite-State Grammar. Front. Psychol. 12:772291.

doi: 10.3389/fpsyg.2021.772291 units repeated in each series generated, as in the sequence $\mathrm{A}^{\mathrm{n}} \mathrm{B}^{\mathrm{n}}$, where the number of A's matches the number of B's (Balari et al., 2011; Longa, 2013). The non-adjacent relations in PSGs are made possible by hierarchical structures that relate items at a distance. Since PSGs require hierarchical structure, $\mathrm{F} \& \mathrm{H}$ conclude that while humans can generate them, tamarins cannot, thus limiting their system to sequences based strictly on adjacent dependency, that is, FSG. There is no doubt that human language requires a grammar more powerful than FSG (Chomsky, 1956, 1959). In this article, I will take up F\&H's assumption that their experiment showed that the tamarin monkeys are capable of learning sequences based on FSG. While their stimuli appear to approximate an FSG, in reality they do not, except trivially. Hence, their conclusion that tamarins are capable of FSG is at best weak. This casts doubt on using the Chomsky hierarchy for describing the learning behavior of nonhuman primates. Furthermore, unlike humans, who are exposed continuously to natural speech that requires a grammar more powerful than FSG, monkeys in nature are never exposed to verbal behavior that reflects FSG in any meaningful sense. It would therefore be surprising if they exhibit mastery of FSG combinations, which are entirely outside their natural experience.

\section{FITCH AND HAUSER'S EXPERIMENTS}

In F\&H's experiments, the stimuli were composed of two categories: in one category are female utterances artificially synthesized into discrete consonant-vowel syllables ( $\boldsymbol{p a}, \boldsymbol{l i}, \boldsymbol{m o}, \boldsymbol{n u}, \boldsymbol{k a} \ldots$. ), and in the other are male utterances similarly synthesized into discrete syllables that differed from the female syllables ( $b a, d i, y o, t u, n o \ldots)$. The male/female syllables also differ distinctly in pitch as well as in other acoustic variables. For FSG, a syllable from one category (A) is followed by a syllable from the other category (B) (e.g., no li). Similar A-B combinations with different syllables were played in sequence, A-B, A-B, A-B. This is a straightforward Markovian system in which a given automaton is carried from one finite state $[\mathrm{n}]$ to the next state $[\mathrm{n}+1]$. F\&H demonstrated that cotton-top tamarin monkeys can learn $(\mathrm{AB})^{\mathrm{n}}$. For PSG, three syllables from one category were played, followed by three syllables from the other category: A-A-A-B-B-B. FSG cannot generate this structure 
without incurring significant cost because the operation depends on non-adjacent information. As F\&H note, the first " $A$ " predicts the occurrence of the final " $B$ ", and the second " $A$ " predicts the second " $\mathrm{B}$ ", and the final " $\mathrm{A}$ " predicts the first " $\mathrm{B}$." This combination reflects a formal grammar higher on the Chomsky hierarchy (Chomsky, 1956)_PSG, which requires hierarchical relations. The tamarin's ability to learn fails completely when presented with the sequence $\mathrm{A}^{\mathrm{n}} \mathrm{B}^{\mathrm{n}}$ generated by PSG. On the other hand, humans readily learn both types of sequences. $\mathrm{F} \& \mathrm{H}$ conclude that the crucial difference is that while humans can generate hierarchical structures that can create non-adjacent dependencies, tamarins are unable to do so, thus limiting their system to adjacent relations. This is an important study for distinguishing human and nonhuman primate learning abilities. The question is, what precisely is the difference? While humans are capable of learning combinations based on a formal grammar more powerful than FSG, I will take issue with F\&H's assumption that what we see with tamarins is an ability to learn combinations based on FSG. ${ }^{1}$

Their so-called FSG is the binary combination, AB. This is FSG only trivially. As I will show, in nature, monkeys are exposed most commonly to a combination of one, but we do see instances of a dual combination. The point is that we don't see anything that exceeds two, which would be surprising if the monkeys are capable of FSG. ${ }^{2}$

\section{QUESTIONING WHETHER TAMARINS CAN LEARN COMBINATIONS BASED ON FSG}

In natural settings, nonhuman primate calls are typically isolated units. The alarm calls of the vervet monkey (Struhsaker, 1967; Seyfarth et al., 1980a,b) is one such system. Vervet monkeys (Chlorocebus pygerythrus) give a distinct call when they encounter a leopard, another when they see an eagle, and a third when they come across a snake. They never combine two calls to produce a new call. This is what Miyagawa and Clarke (2019) call the System of One, and it is the predominant system for alarm calls. This leads to the question, what do we make of the demonstrated ability of tamarins to be able to learn A-B sequences?

\section{SYSTEM OF TWO}

Some Old World monkeys such as the Guenons of Africa produce utterances that Miyagawa and Clarke (2019), based on much prior research, analyzed as being composed of two items. A key

\footnotetext{
${ }^{1}$ Studies have attempted to demonstrate that humans are not the only ones capable of mastering grammars above FSG. These include Rey et al. (2012) for baboons, Abe and Watanabe (2011) for Bengalese finches, and Gentner et al. (2006) for starlings. See Fitch (2017) and ten Cate (2017) for critiques. There are other works that have tested whether nonhuman animals are capable of learning non-adjacent dependencies, including Newport and Aslin (2004), Newport et al. (2004), Malassis et al. (2018), and Versace et al. (2019). As Wilson et al. [(2020): 853] conclude, "these studies demonstrate that at least some nonhuman animals appear to be sensitive to these types of nonadjacent dependencies, but also point to potential cross-species differences, including between humans and nonhuman animals, in how they might be learned."

${ }^{2}$ See Rendall (2021) for critique of recent work on primate semantics and syntax.
}

observation is that this binary system is just that-binary. One never sees a combination that begins $\mathrm{A}, \mathrm{B}$, then goes to $\mathrm{C}$, or returns to $\mathrm{A}$. This is a fundamentally different behavior from what $\mathrm{F} \& \mathrm{H}$ would predict, because a sequence of $\mathrm{A}-\mathrm{B}-\mathrm{C}$ or $\mathrm{A}-$ $\mathrm{B}-\mathrm{A}$ is possible in FSG. Below, I will demonstrate this binary nature using the system employed by putty-nosed monkeys (Cercopithecus nictitans).

There are two main alarm calls associated with the puttynosed monkeys, pyow $(=\mathrm{P})$, which is a general alarm call, and hack $(=\mathrm{H})$, which is typically used in the presence of eagles. The putty-nosed monkeys also produce pyow-hack combinations consisting of a number of pyows followed by a number of hacks. While the individual pyows and hacks are alarm calls, the pyow-hack sequences relate to group movement. Using playback experiments, Arnold and Zuberbühler (2006a,b, 2008, 2012, 2013) demonstrated that the overall length of the sequence is statistically related to the distance traveled by the group; the number of pyows and hacks within the equal-length sequences did not affect the distance. Thus, the researchers observed similar behavior when they played back $\mathrm{PPPHHH}, \mathrm{PHHHHH}$, and other $\mathrm{P}-\mathrm{H}$ combinations of the same length.

Schlenker et al. [(2016): 33] point out that the different pyowhack sequences of the same length are phonologically complex, but lexically simple. They are phonologically complex due to the various numbers of pyows and hacks (see also Mitani and Marler, 1989). The sequences are lexically simple because they are associated with comparable distance traveled, regardless of the number of actual pyows and hacks. How can we capture both the phonological complexity and the lexical simplicity of these sequences? Looking at the different possibilities, there are two compartments, one for pyows, the other for hacks, as shown in Table 1.

Each compartment may contain a varying number of pyows or a varying number of hacks. ${ }^{3}$ Crucially, we never find a sequence such as PHP (Arnold and Zuberbühler, 2012), because this sequence would require more than two compartments. On the FSG view of monkey learning behavior, we would predict that PHP is possible, contrary to fact. ${ }^{4}$

Other systems that Miyagawa and Clarke (2019) explore have the same dual-compartment character. The dual-compartment

TABLE 1 | Dual-compartment frame (Miyagawa and Clarke, 2019).

\begin{tabular}{cc} 
pyow $^{+}$ & hack $^{+}$ \\
\hline 1 & 2
\end{tabular}

\footnotetext{
${ }^{3}$ Progovac (2015) proposed what she calls a "two-slot mold." This is similar to our dual-compartment frame, although her proposal is based on speculation about earlier forms of human language.

${ }^{4}$ Most works aiming at eliciting an FSG grammar from nonhuman primates resort to pattern discrimination rather than on the combinatorial complexity of vocal production. There is no known case of vocal production that combines more than two calls, as observed in Miyagawa and Clarke (2019). This binarity recalls the dichotomy between vocal production/vocal perception in nonhuman primates: while the former is highly restricted, the latter is argued to be more sophisticated (Seyfarth and Cheney, 1986, 2003; Fitch and Zuberbühler, 2013). At this point, however, there is no reason to associate a full-fledged FSG to tamarins even in their pattern-discrimination perception.
} 
frame can trivially be modeled by FSG, but it is by no means FSG in the standard sense in that there is no operation of any kind that can potentially lead to strings of infinite length.

\section{DISCUSSION}

While $\mathrm{F} \& \mathrm{H}$ used the $(\mathrm{AB})^{\mathrm{n}}$ stimulus under the assumption that this models FSG, I suggest that what F\&H demonstrated for tamarins was that they are capable of learning binary combinations, which occur in natural settings. In fact, F\&H [(2004): 379] entertain the possibility that "tamarins fail the PSG because their ability to differentiate successive items is limited to runs of two." They reject this idea because they tested A-A$\mathrm{B}-\mathrm{B}$ along with $\mathrm{A}-\mathrm{A}-\mathrm{A}-\mathrm{B}-\mathrm{B}-\mathrm{B}$, and tamarins failed to learn both sequences. However, A-A-B-B sequence cannot easily fit into the dual-compartment frame because for each $\mathrm{A}$, there is $\mathrm{B}$. This kind of relation is expressed by a hierarchical structure, as F\&H themselves note. This, in turn, casts doubt on applying formal grammar based on the Chomsky hierarchy for distinguishing learning behavior of nonhuman primates from that of humans. The learning behavior of nonhuman primates does not appear susceptible even to the simplest formal grammar (FSG) on the hierarchy.

There is also neuroanatomical evidence for the idea that the $(\mathrm{AB})^{\mathrm{n}}$ sequence as used by $\mathrm{F} \& \mathrm{H}$ does not implicate FSG. Friederici et al. (2006) (see also Friederici et al., 2012) demonstrated that the PSG sequence, $A^{n} B^{n}$, similar to the stimulus created by $\mathrm{F} \& \mathrm{H}$, activates Brodmann area 44 of the Broca's area and the frontal operculum. ${ }^{5}$ In contrast, the "FSG" sequence of $(A B)^{n}$ only recruits the frontal operculum. The frontal operculum is a phylogenetically older part of the brain

\footnotetext{
${ }^{5}$ Kaan and Swaab (2002) and Matchin and Hickok (2016) argue that syntactic operations do not just recruit a specific frontal region such as the Broca's.
}

than the Broca's area (Sanides, 1962). As Zaccarella and Friederici (2015a,b,c) note, one of its functions is apparently to create (AB) combinations, which we see in monkeys (Sanides, 1962) and in humans. ${ }^{6}$ The Broca's area and the frontal operculum each has a unique functional, anatomical and molecular brain architecture (Sanides, 1962; Amunts et al., 1999, 2010; Zilles and Amunts, 2009). It is Broca's region of the brain that is recruited for the complex PSG-based sequence, which has a hierarchical structure. ${ }^{7}$ From this perspective, the $\mathrm{AB}$ sequence that F\&H showed to be learnable by tamarins need not be understood as an indication of their ability to learn combinations based on FSG. Rather, it fits the binary combination that models the dual-compartment frame arguably activated in the frontal operculum.

\section{AUTHOR CONTRIBUTIONS}

The author confirms being the sole contributor of this work and has approved it for publication.

\section{ACKNOWLEDGMENTS}

I am grateful to the reviewer for numerous helpful suggestions, as well as to the associate editor and Vitor Nóbrega for comments that helped to clarify many of the points.

${ }^{6}$ In Zaccarella and Friederici (2015a,b,c), they assign to the adjacent anterior insula the role of processing two-word sequences, a brain region that is linked to the frontal operculum [(Friederici, 2017): 40-42].

${ }^{7}$ Other studies implicate the left anterior temporal lobe in human language combinatorial/hierarchical operations without mention of Broca's area and the frontal operculum (Bemis and Pylkkänen, 2011; Brennan and Pylkkänen, 2017).

\section{REFERENCES}

Abe, K., and Watanabe, D. (2011). Songbirds possess the spontaneous ability to discriminate syntactic rules. Nat. Neurosci. 14, 1067-1074. doi: 10.1038/nn.2869

Amunts, K., Lenzen, M., Friederici, A. D., Schleicher, A., Morosan, P., Palomero-Gallagher, N., et al. (2010). Broca's region: novel organizational principles and multiple receptor mapping. PLoS Biol. 8:e1000489. doi: 10.1371/journal.pbio.1000489

Amunts, K., Schleicher, A., Bürgel, U., Mohlberg, H., Uylings, H. B., and Zilles, K. (1999). Broca's region revisited: cytoarchitecture and intersubject variability. J. Comp. Neurol. 412, 319-341. doi: 10.1002/(SICI)10969861(19990920)412:2<319::AID-CNE10>3.0.CO;2-7

Arnold, K., and Zuberbühler, K. (2006a). Language evolution: semantic combinations in primate calls. Nature. 441, 303-303. doi: 10.1038/441303a

Arnold, K., and Zuberbühler, K. (2006b). The alarm-calling system of adult male putty-nosed monkeys, Cercopithecus nictitans martini. Anim. Behav. 72, 643-653. doi: 10.1016/j.anbehav.2005.11.017

Arnold, K., and Zuberbühler, K. (2008). Meaningful call combinations in a nonhuman primate. Curr. Biol. 18, R202-R203. doi: 10.1016/.j.cub.2008.01.040

Arnold, K., and Zuberbühler, K. (2012). Call combinations in monkeys: compositional or idiomatic expressions? Brain Lang. 120, 303-309. doi: 10.1016/j.bandl.2011.10.001

Arnold, K., and Zuberbühler, K. (2013). Female putty-nosed monkeys use experimentally altered contextual information to disambiguate the cause

of male alarm calls. PLoS ONE. 8:e65660. doi: 10.1371/journal.pone. 0065660

Balari, S., Benítez-Burraco, A., Camps, M., Longa, V. M., Lorenzo, G., and Uriagereka, J. (2011). The archaeological record speaks: Bridging anthropology and linguistics. Int. J. Dev. Biol. 11, 1-17. doi: 10.4061/2011/382679

Bemis, D. K., and Pylkkänen, L. (2011). Simple composition: a magnetoencephalography investigation into the comprehension of minimal linguistic phrases. J. Neurosci. 31, 2801-2814. doi: 10.1523/ JNEUROSCI.5003-10.2011

Brennan, J. R., and Pylkkänen, L. (2017). MEG evidence for incremental sentence composition in the anterior temporal lobe. Cogn. Sci. 41, 1515-1531. doi: 10.1111/cogs.12445

Chomsky, N. (1956). Three models for the description of language. IEEE Trans. Inf. Theory. 2, 113-124. doi: 10.1109/TIT.1956.1056813

Chomsky, N. (1959). On certain formal properties of grammars. Inf. Control. 2, 137-167. doi: 10.1016/S0019-9958(59)90362-6

Fitch, W. T. (2017). "Dendrophilia and the Evolution of Syntax," in Origins of Human Language: Continuities and Discontinuities with Nonhuman Primates, eds. L-J. Boë, J. Fagot, P. Perrier, and J-L. Schwartz (Bern: Peter Lang), 305-328.

Fitch, W. T., and Hauser, M. D. (2004). Computational constraints on syntactic processing in a nonhuman primate. Science. 303, 377-380. doi: 10.1126/science.1089401

Fitch, W. T., and Zuberbühler, K. (2013). "Primate precursors to human language: Beyond discontinuity," in Evolution of Emotional Communication: From Sounds in Nonhuman Mammals to Speech and Music in Man, eds. E. Altenmüller, 
S. Schmidt, and E. Zimmermann (Oxford: Oxford University Press) 26-48. doi: 10.1093/acprof:oso/9780199583560.003.0002

Friederici, A. (2017). Language in our brain: The origins of a uniquely human capacity. Cambridge: MIT Press. doi: 10.7551/ mitpress/9780262036924.001.0001

Friederici, A. D., Bahlmann, J., Heim, S., Schubotz, R. I., and Anwander, A. (2006). The brain differentiates human and non-human grammars: functional localization and structural connectivity. Proc. Natl. Acad. Sci. USA. 103, 2458-2463. doi: 10.1073/pnas.0509389103

Friederici, A. D., Oberecker, R., and Brauer, J. (2012). Neurophysiological preconditions of syntax acquisition. Psychol. Res. 76, 204-211. doi: $10.1007 / \mathrm{s} 00426-011-0357-0$

Gentner, T., Fenn, K., Margoliash, D., and Nusbaum, H. C. (2006). Recursive syntactic pattern learning by songbirds. Nature. 440, 1204-1207. doi: 10.1038/nature04675

Kaan, E., and Swaab, T. Y. (2002). The brain circuitry of syntactic comprehension. Trends Cogn. Sci. 6, 350-356. doi: 10.1016/S1364-6613(02)01947-2

Longa, V. M. (2013). The evolution of the Faculty of Language from a Chomskyan perspective: Bridging Linguistics and Biology. J Anthropol Sci. 91, 1-48. doi: 10.4436/JASS.91011

Malassis, R., Rey, A., and Fagot, J. (2018). Non-adjacent dependencies processing in human and non-human primates. Cognitive Sci. 42, 1677-1699. doi: $10.1111 /$ cogs. 12617

Matchin, W., and Hickok, G. (2016). 'Syntactic perturbation' during production activates the right IFG, but not Broca's area or the ATL. Front. Psychol. 7:241. doi: $10.3389 /$ fpsyg. 2016.00241

Mitani, J. C., and Marler, P. (1989). A phonological analysis of male gibbon singing behavior Behaviour 109, 20-45. doi: 10.1163/156853989X00141

Miyagawa, S., and Clarke, E. (2019). Systems underlying human and old world monkey communication: one, two, or infinite. Front. Psychol. 10:1911. doi: 10.3389/fpsyg.2019.01911

Newport, E. L., and Aslin, R. N. (2004). Learning at a distance I. Statistical learning of non-adjacent dependencies. Cognitive Psychol. 48, 127-162. doi: 10.1016/S0010-0285(03)00128-2

Newport, E. L., Hauser, M. D., Spaepen, G., and Aslin, R. N. (2004). Learning at a distance II. Statistical learning of non-adjacent dependencies in a non-human primate. Cognitive Psychol. 49, 85-117. doi: 10.1016/j.cogpsych.2003.12.002

Progovac, L. (2015). Evolutionary syntax. Oxford: Oxford University Press. doi: 10.1093/acprof:oso/9780198736547.001.0001

Rendall, D. (2021). Aping language: Historical perspectives on the quest for semantics, syntax, and other rarefied properties of human language in the communication of primates and other animals. Front. Psychol. 12:675172. doi: $10.3389 /$ fpsyg.2021.675172

Rey, A., Perruchet, P., and Fagot, J. (2012). Centre-embedded structures are a by-product of associative learning and working memory constraints: Evidence from baboons (Papio Papio). Cognition. 123, 180-184. doi: 10.1016/j.cognition.2011.12.005

Sanides, F. (1962). Die Architektonik des Menschlichen Stirnhirns: Zugleich eine Darstellung der Prinzipien Seiner Gestaltung als Spiegel der Stammesgeschichtlichen Differenzierung der Grosshirnrinde. Berlin/Heidelberg: Springer-Verlag. Available online at: www.springer.com/de/book/9783540028864 (accessed in September 6, 2021). doi: 10.1007/978-3-642-86210-6

Schlenker, P., Chemla, E., Schel, A. M., Fuller, J., Gautier, J.-P., Kuhn, J., et al. (2016). Formal monkey linguistics. Theor. Linguist. 42, 1-90. doi: 10.1515/tl-2016-0001
Seyfarth, R., Cheney, D., and Marler, P. (1980a). Monkey responses to three different alarm calls: evidence of predator classification and semantic communication. Science. 210, 801-803. doi: 10.1126/science.74 33999

Seyfarth, R. M., and Cheney, D. L. (1986). Vocal development in vervet monkeys. Anim. Behav. 34, 1640-1658. doi: 10.1016/S0003-3472(86)8 0252-4

Seyfarth, R. M., and Cheney, D. L. (2003). Signalers and receivers in animal communication. Annu. Rev. Psychol. 54, 145-173. doi: 10.1146/annurev.psych.54.101601.145121

Seyfarth, R. M., Cheney, D. L., and Marler, P. (1980b). Vervet monkey alarm calls: semantic communication in a free-ranging primate. Anim. Behav. 28, 1070-1094. doi: 10.1016/S0003-3472(80)80097-2

Struhsaker, T. T. (1967). "Auditory communication among vervet monkeys (Cercopithecus aethiops)," in Social communication among primates, ed. S. A. Altman (Chicago: University of Chicago Press) 281-324.

ten Cate, C. (2017). Assessing the uniqueness of language: Animal grammatical abilities take center stage. Psychon. Bull. Rev. 24, 91-96. doi: 10.3758/s13423-016-1091-9

Versace, E., Rogge, J. R., Shelton-May, N., and Ravignani, A. (2019). Positional encoding in cotton-top tamarins (Saguinus oedipus). Anim. Cogn. 22, 281-324. doi: 10.1007/s10071-019-01277-y

Wilson, B., Spierings, M., Ravignani, A., Mueller, J. L., and Mintz, T. H., Frank et al. (2020). Non-adjacent dependency learning in humans and other animals. Top Cogn Sci. 12, 843-858. doi: 10.1111/tops.12381

Zaccarella, E., and Friederici, A. D. (2015a). Reflections of word processing in the insular cortex: a sub-regional parcellation based functional assessment. Brain Lang. 142, 1-7. doi: 10.1016/j.bandl.2014.12.006

Zaccarella, E., and Friederici, A. D. (2015b). Merge in the Human Brain: A SubRegion Based Functional Investigation in the Left Pars Opercularis. Front. Psychol. 6:1818. doi: 10.3389/fpsyg.2015.01818

Zaccarella, E., and Friederici, A. D. (2015c). "Syntax in the Brain," in Brain Mapping: An Encyclopedic Reference, ed. Arthur W. Toga (Academic Press: Elsevier) 461-468. doi: 10.1016/B978-0-12-397025-1. 00268-2

Zilles, K., and Amunts, K. (2009). Receptor mapping: architecture of the human cerebral cortex. Curr. Opin. Neurol. 22, 331-339. doi: 10.1097/WCO.0b013e32832d95db

Conflict of Interest: The author declares that the research was conducted in the absence of any commercial or financial relationships that could be construed as a potential conflict of interest.

Publisher's Note: All claims expressed in this article are solely those of the authors and do not necessarily represent those of their affiliated organizations, or those of the publisher, the editors and the reviewers. Any product that may be evaluated in this article, or claim that may be made by its manufacturer, is not guaranteed or endorsed by the publisher.

Copyright (c) 2021 Miyagawa. This is an open-access article distributed under the terms of the Creative Commons Attribution License (CC BY). The use, distribution or reproduction in other forums is permitted, provided the original author(s) and the copyright owner(s) are credited and that the original publication in this journal is cited, in accordance with accepted academic practice. No use, distribution or reproduction is permitted which does not comply with these terms. 